\footnotetext{
*Corresponding autor

Email address: kotov@bnl.gov (I. V. Kotov)

Preprint submitted to Elsevier
} 
tool for trap identification algorithms development. The pocket pumped image for segment 11 is shown in Fig.1, as viewed in ds9 and zoomed on the area with large trap density.

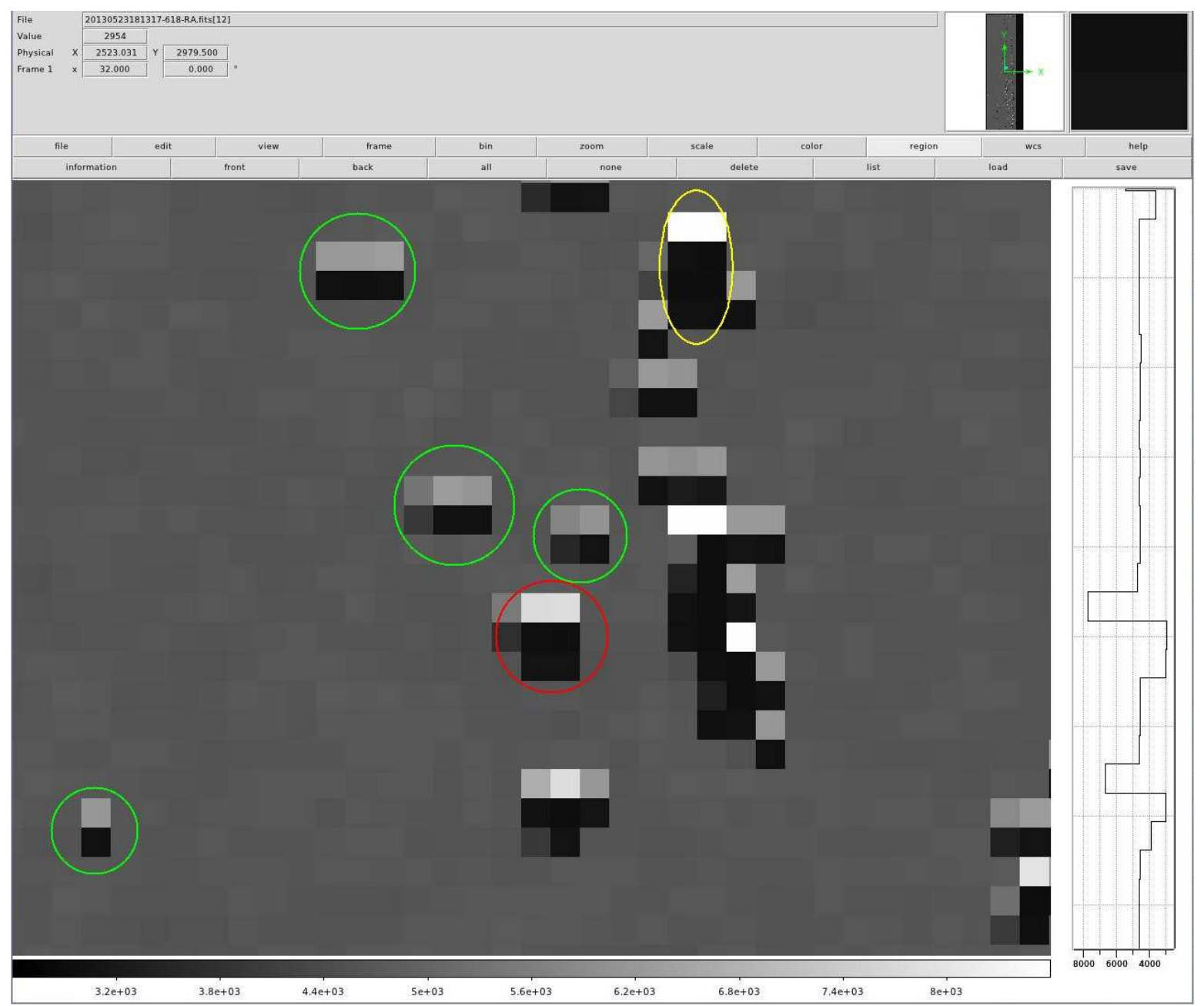

Figure 1: Segment 11 high trap density area as viewed in ds9. The column direction is Up.

\section{Initial Image Processing}

As a first step of image processing the average overscan amplitude has been subtracted for each row. A histogram for the segment 12 image is shown in Fig.2. Beside the primary flat field peak several other

gone. The primary peak is positioned at zero which is convenient for further analysis. 

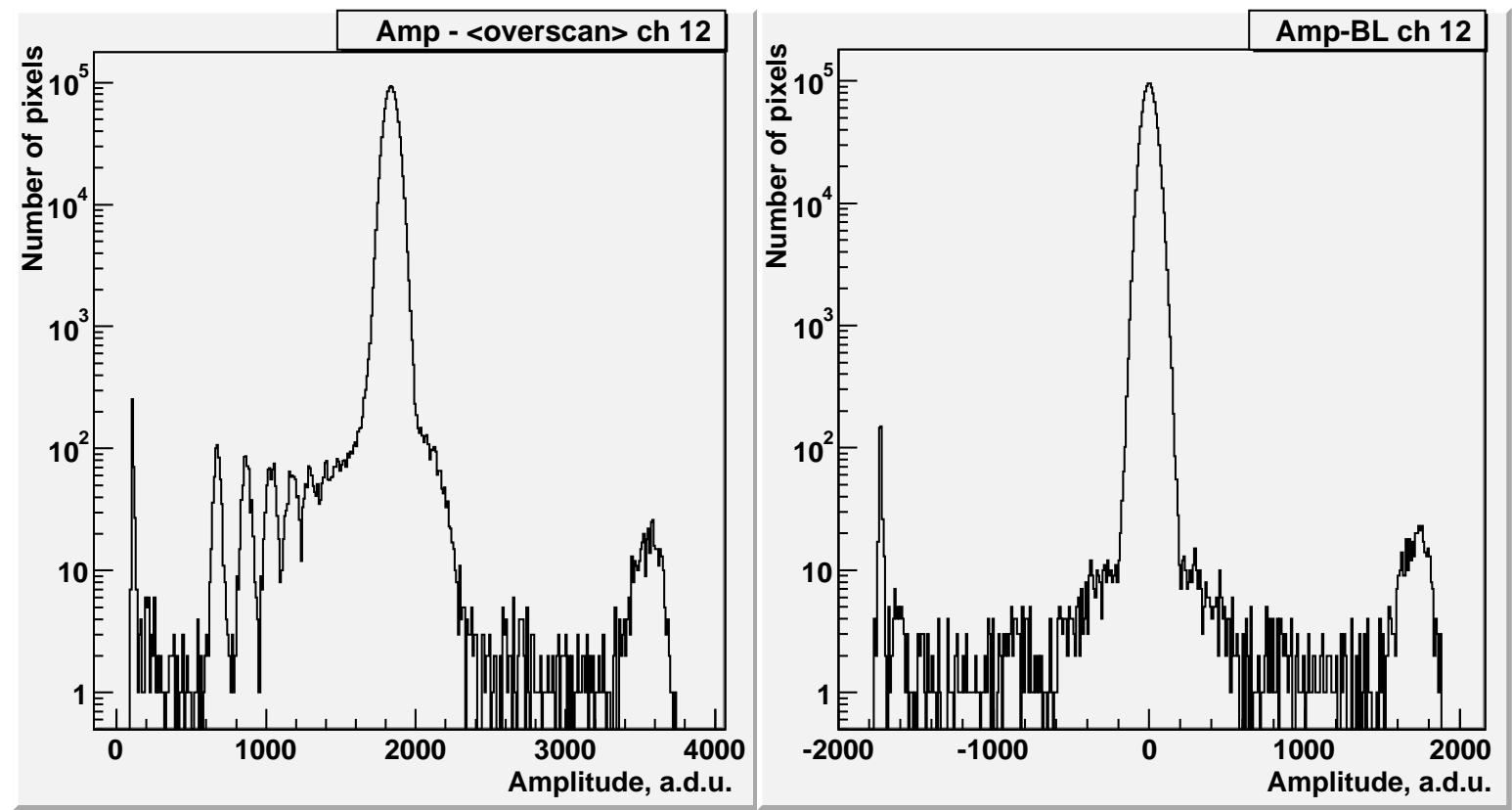

Figure 2: The segment 12 histogram before and after the Base Line subtraction.

\section{Trap Identification. Intuitive way.}

A trap causes negative correlation between adjacent pixel amplitudes along the column direction. One way to see this correlation is to look at the amplitude scatter plot for neighboring pixel pairs. Scatter plots for different cuts are shown in Fig.3. It should be noted that each pixel participates in 2 points on the scatter plot Fig.3.b. Ones when it is the first pixel of the pair and second time when it is the second pixel.

The negative correlation caused by traps is clearly present. Points are concentrated along the line with slope -1, the trap band. In a simple situation of isolated traps, low trap density case, only one data point belongs to the dipole signal. Pairs with one pixel from the dipole signal and another from outside of the dipole form "arms of the cross", vertical and horizontal bands of points. Simple cut was applied to amplitudes to eliminate these features. Pairs with amplitude $<3 \sigma$ of the shot noise were excluded. This cut results in the scatter plots shown in Fig.3.c,d. Even after amplitude cut is applied points are present outside the trap band. For comparison let's look into a segment 12 where the trap concentration is lower, corresponding plots are shown in Fig.4.

The trap band is practically the only feature left after the amplitude cuts have been applied as seen in Fig.4d plot. This comparison clearly demonstrates that on Fig.3 point concentration in the low left corner, both amplitudes are low, and the band parallel to the trap band are related to the high trap density observed in some regions.

One of the regions with the high trap density is shown in Fig.1 and some hints can be extracted from this 

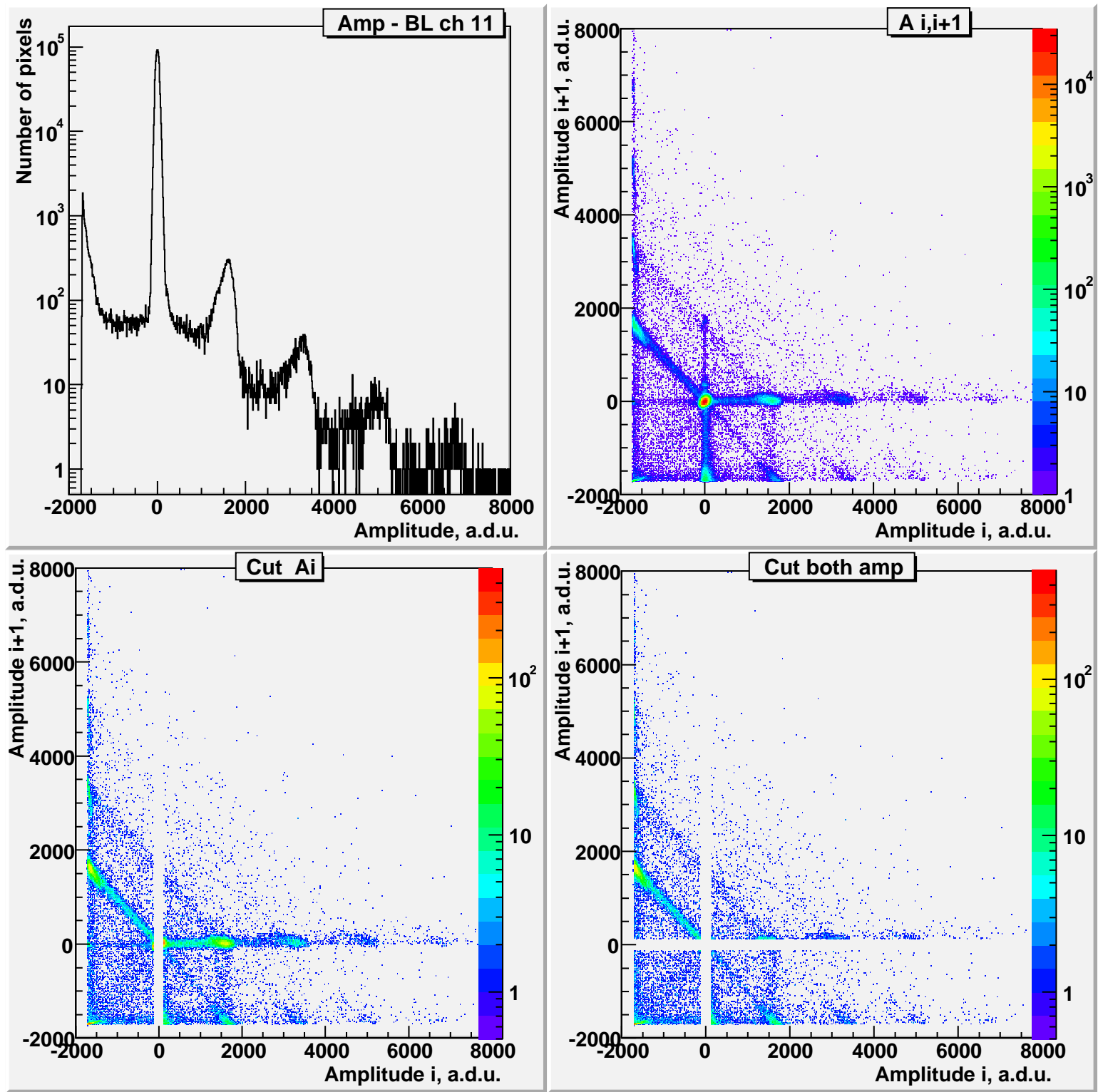

Figure 3: Segment 11 plots: a) the histogram (upper left panel); b) neighboring pixel amplitudes scatter plot (upper right panel); c, d) scatter plot with cuts applied (bottom panels). 

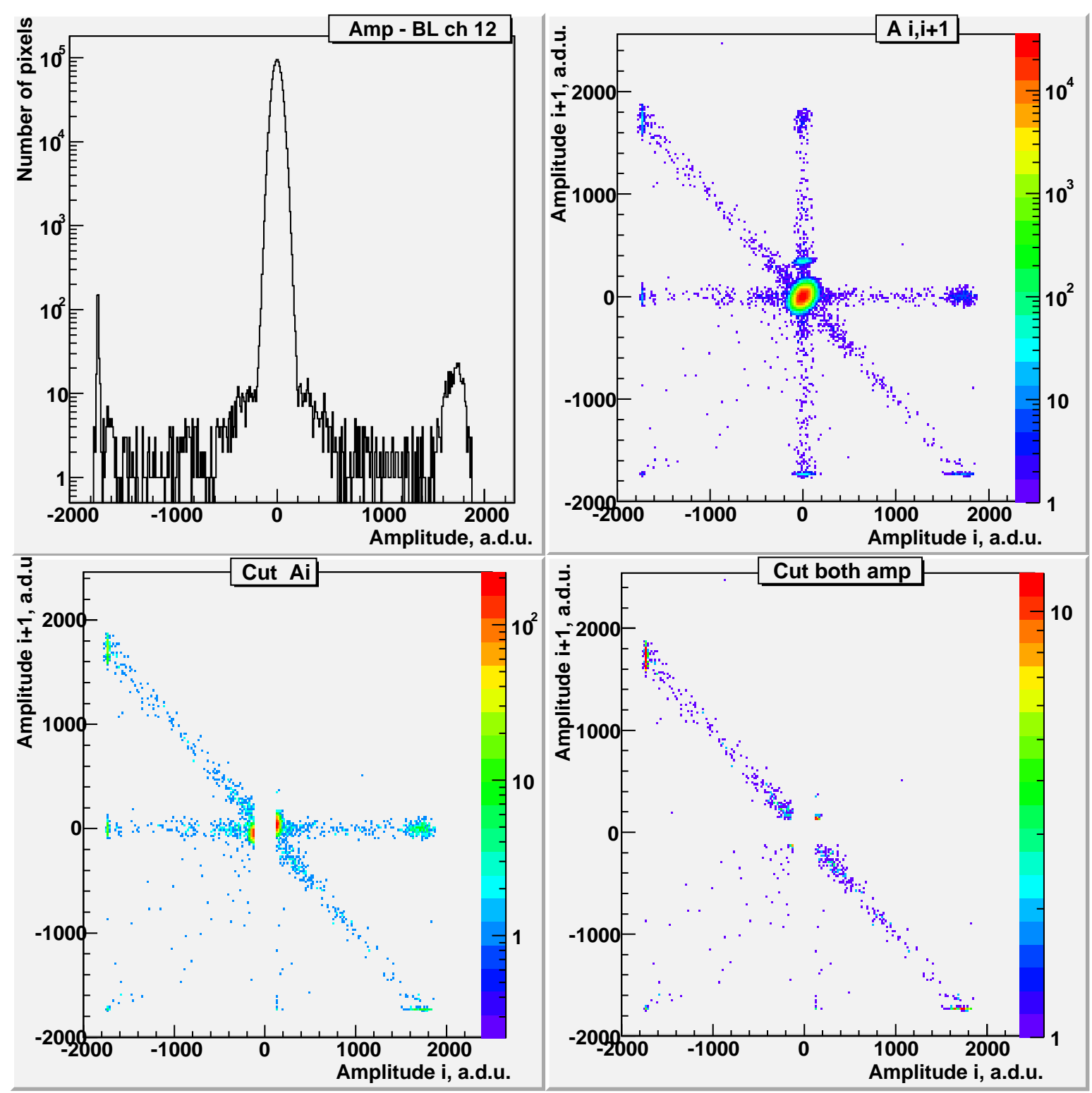

Figure 4: Segment 12 plots: a) the histogram (upper left panel); b) neighboring pixel amplitudes scatter plot (upper right panel); c, d) scatter plot with cuts applied (bottom panels). 
image. The color of the region stands for: green - isolated traps, red - "triplets", yellow - "quadruplet". The triplets are formed when there are enough traps to facilitate pumping from 2 pixels into one $(i \rightarrow i+1 \rightarrow$ $i+2)$ and quadruplets when charge is pumped from 3 pixels into one. There are even multiplets extended to more than 4 pixels in segment 11. For such long transfers to happen more than one trap should be present in a single pixel. For example, middle pixel of the triplet should have at least 2 traps. In Fig.3, triplets are responsible for the band parallel to the trap band and also for points in the region where both amplitudes are low (2 depleted pixels of a triplet or longer multiplet). Unambiguous trap counting and positioning is possible only for isolated traps. The trap concentration in good quality segments is low and probability of multiple traps in one pixel is second order effect. Pixels belonging to the isolated trap band can be easily selected, counted and sub-pixel trap location can be determined (clock phase 1-4, see [3]). This can be done using 2D cut around the band in Fig.3d or Fig.4d plots. There are other options and let's look into them.

\section{Trap Identification. Formal way.}

The quantity reflecting the significance of a dipole signal, or in other words significance of the correlation between pixel amplitudes, is the correlation coefficient or correlator. The expression for the correlator is

$$
C_{2}=\frac{A_{i}}{\sigma} \times \frac{A_{i+1}}{\sigma}
$$

where $A_{i}$ is base line subtracted amplitude in the first pixel of the pair, $A_{i+1}$ is base line subtracted amplitude in the second pixel of the pair and $\sigma$ is the sigma of the flat field shot noise. The correlation caused by traps is negative. Correlator plots for segments 12 and 11 are shown in Fig.5 and Fig.6 correspondingly.

The segment 12 with lower trap density beautifully demonstrates this negative correlation coming almost entirely from isolated trap sites. In this case the parabolic shape is expected for amplitude dependence since amount of charge lost in one pixel is equal to amount of charge gained by another pixel and $C_{2} \sim-A^{2}$. Plots of first pixel amplitudes, $A_{i}$ and the number of traps in individual columns are shown in Fig.7 and Fig. 8 for segments 12 and 11 correspondingly. The number of entries in these plots gives the total trap count.

The selection of the trap bands presented in the amplitude scatter plot can be simplified using coordinate system transformation. The useful transformation is rotation by 45 degree in $\left[a m p_{i}, a m p_{i+1}\right]$ plane. The relationship between "rotated" coordinates $[A+, A-]$ and amplitudes is $A+=\left(a m p_{i}+a m p_{i+1}\right) / \sqrt{2}, A-=$ $\left(a m p_{i+1}-a m p_{i}\right) / \sqrt{2}$.

\section{CONCLUSIONS}

The novel trap identification technique has been developed. This technique has been used to analyze pocket pumped images obtained with CCD 250. Using this technique the following information can be 

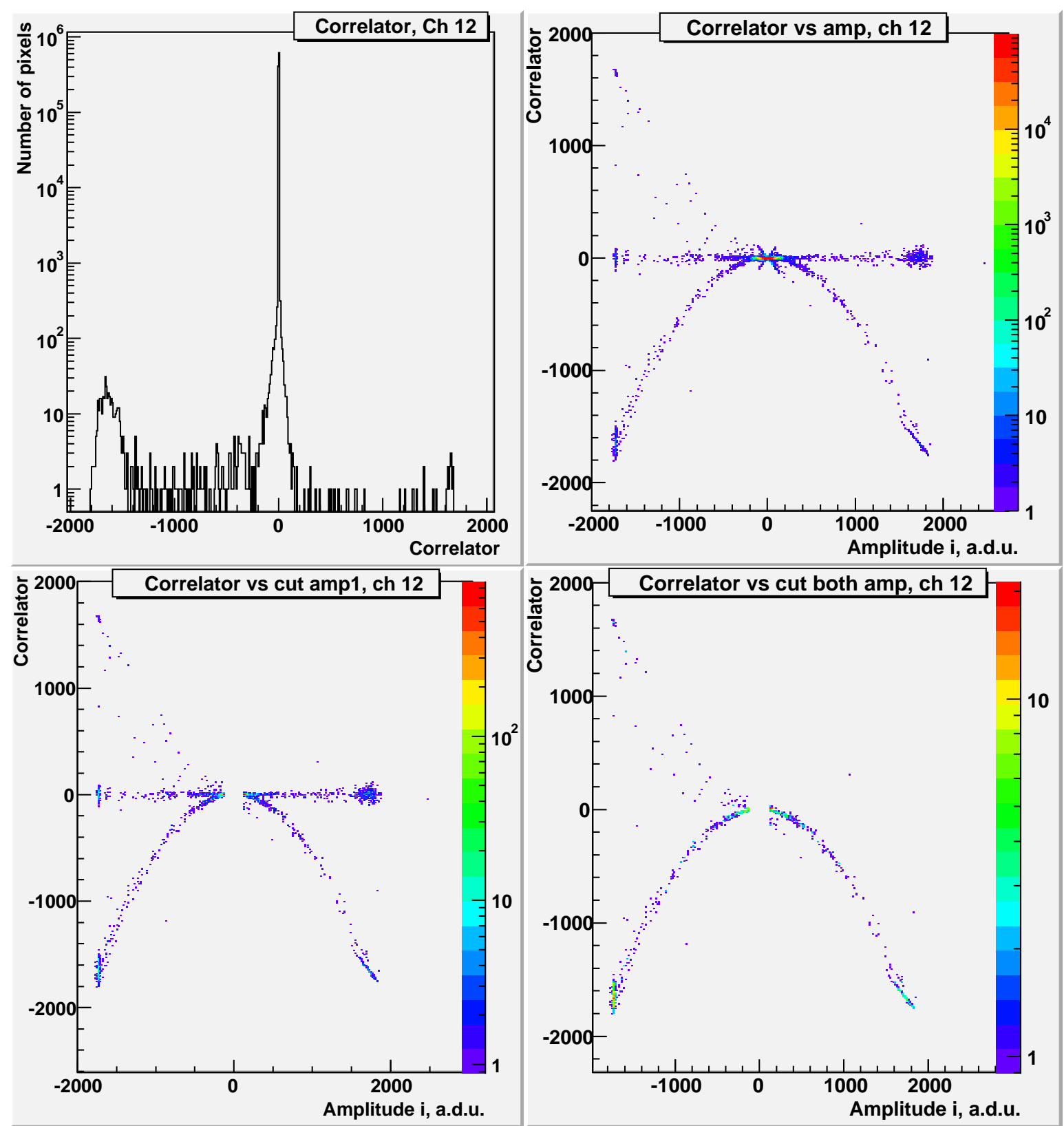

Figure 5: Segment 12 plots: a) the correlator (upper left panel); b) correlator vs first amplitude (upper right panel); c, d) scatter plot with amplitude cuts applied (bottom panels). 

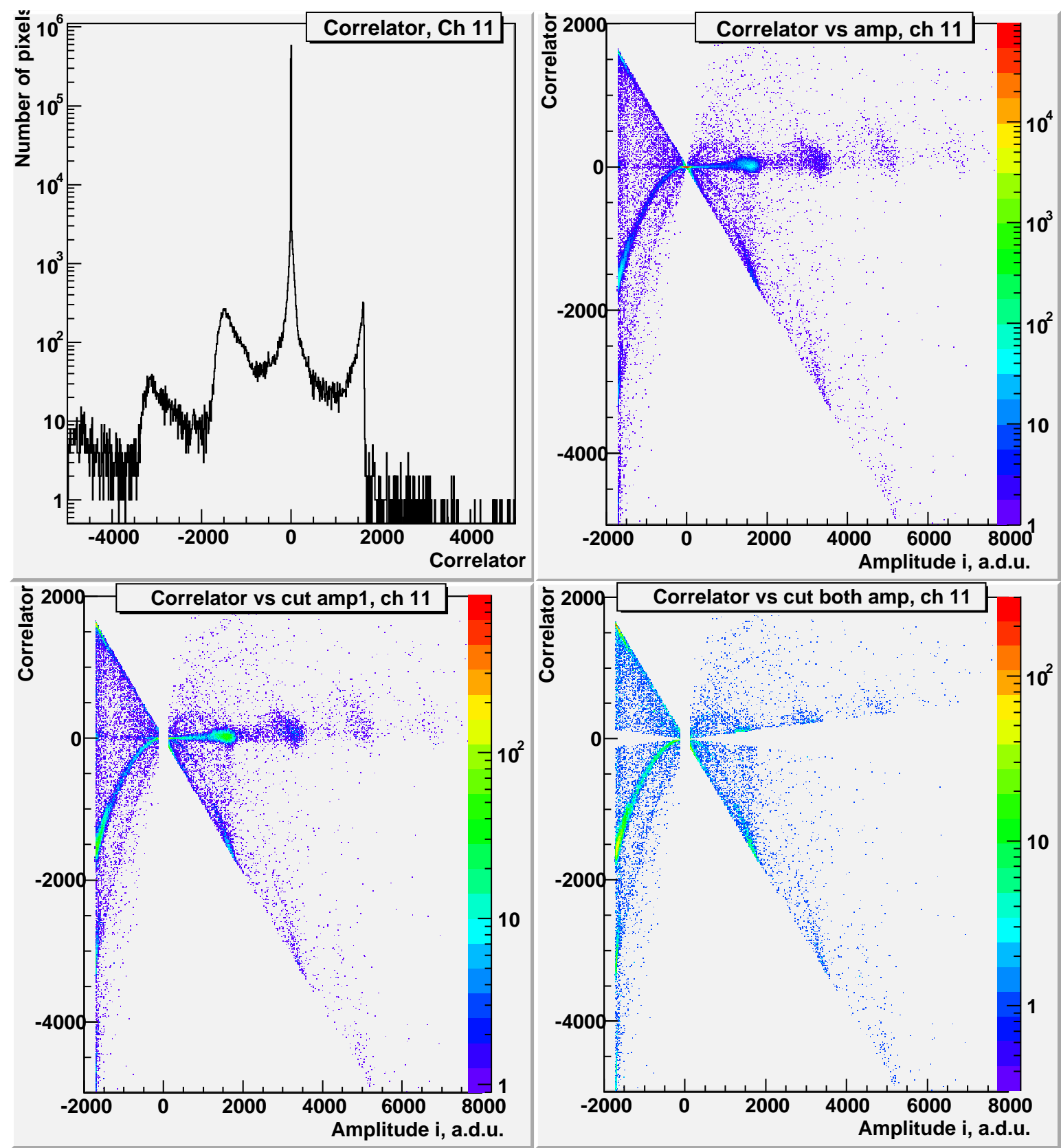

Figure 6: Segment 11 plots: a) the correlator (upper left panel); b) correlator vs first amplitude (upper right panel); c, d) scatter plot with amplitude cuts applied (bottom panels). 


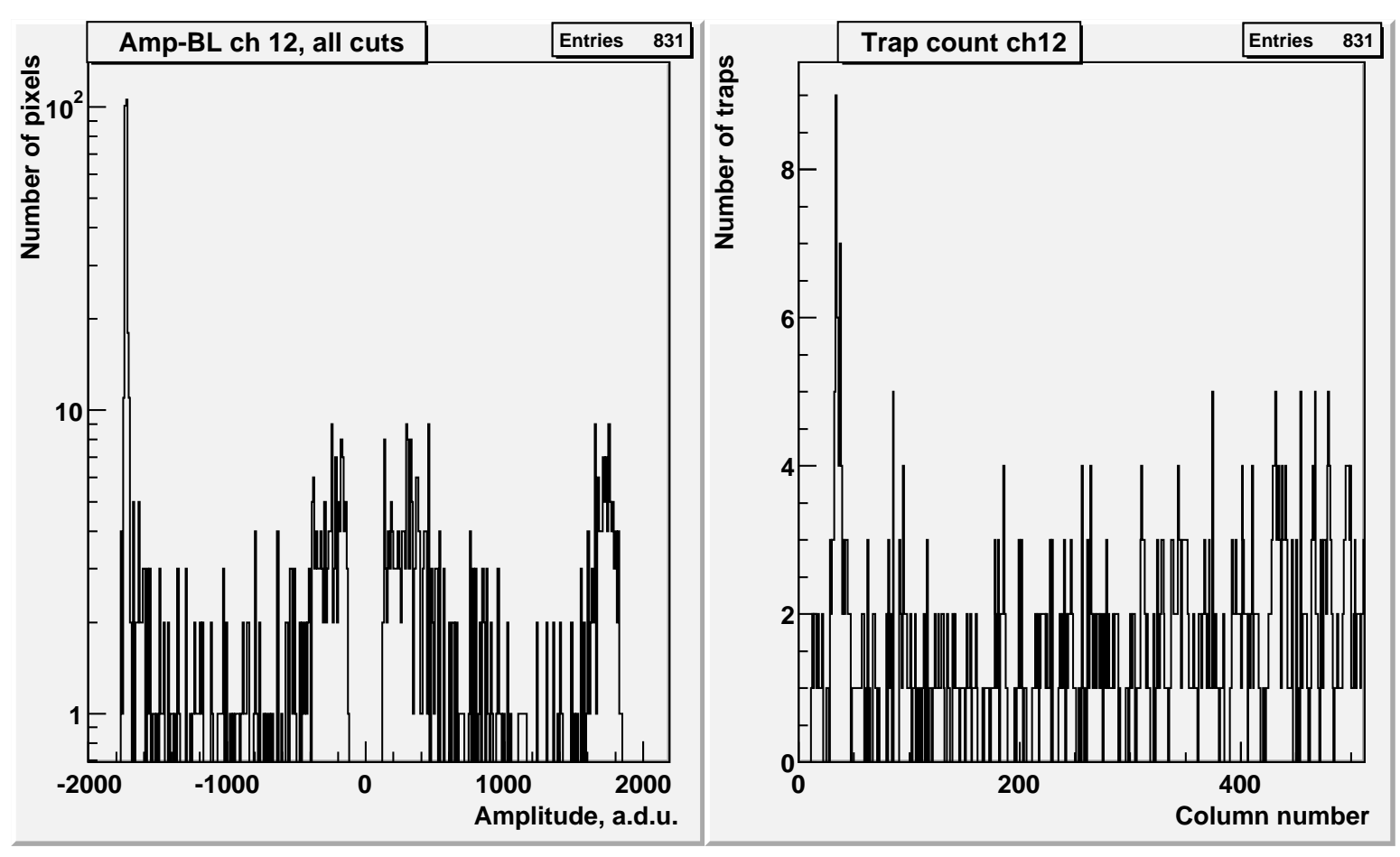

Figure 7: Segment 12 plots: a)first pixel amplitude after all cuts (left panel) and b) trap count in columns (right panel).
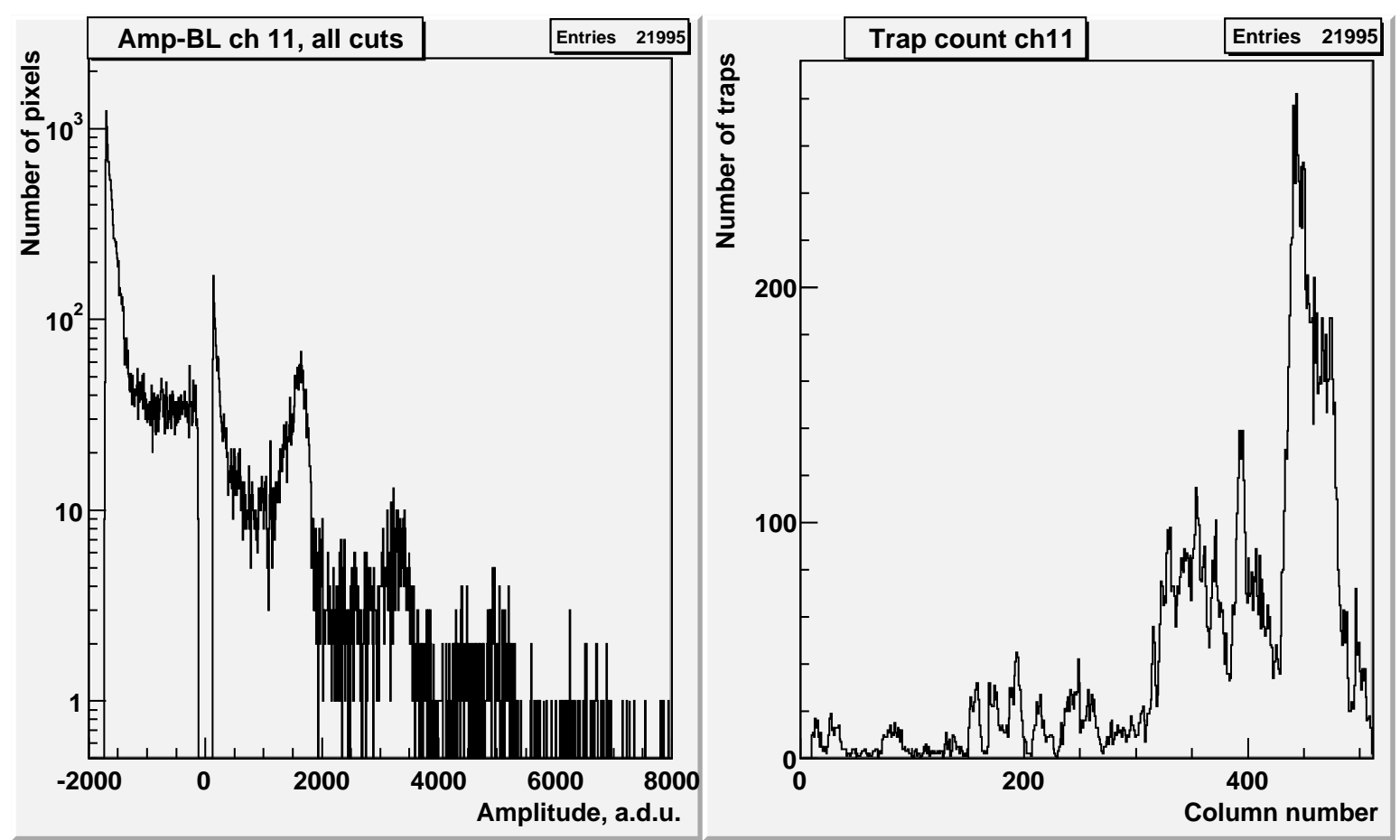

Figure 8: Segment 11 plots: a) first pixel amplitude after all cuts (left panel) and b) trap count in columns (right panel). 
extracted from a single pocket pumped image:

- trap counts: total; per segment; in each column;

- trap locations;

- initial estimate of trap pumping efficiency;

The developed technique allows for counting all traps even traps with low pumping efficiency down to statistically detectable level. This is clearly enables one to extract maximum information from the given data set. In previous studies, for example $[2,5]$, traps were selected only from the efficiently pumped peak (in our case peak at about $1800 \mathrm{adu}$ ) which was enough for their purposes.

The dipole orientation indicates the trap location. The measurements with integration performed under different clock phases can even reveal the trap sub-pixel location as discussed in [3].

The pumping efficiency can be estimated from the amplitude of the pumped signal. Maximum pixel depletion corresponds to hundred percent efficiency and smaller amplitude corresponds to proportionally smaller pumping efficiency. So the pumped signal amplitude is proportional to the trap pumping efficiency. Should be noted that there is pumping efficiency ambiguity for fully pumped out pixels. Additional measurements with different number of pumping cycles or different light exposures are required to separate pixels containing efficient single trap from pixels with multiple traps.

\section{ACKNOWLEDGMENTS}

Authors acknowledge e2v technologies, Semiconductor Technology Associates and Imaging Technology Laboratory help in sensor prototype development.

This manuscript has been co-authored by employees of Brookhaven Science Associates, LLC.

LSST project activities are supported in part by the National Science Foundation through Governing Cooperative Agreement 0809409 managed by the Association of Universities for Research in Astronomy (AURA), and the Department of Energy under contract DE-AC02-76-SFO0515 with the SLAC National Accelerator Laboratory. Additional LSST funding comes from private donations, grants to universities, and in-kind support from LSSTC Institutional Members.

\section{References}

[1] D.Burt, J.Endicott, P.Jerram, P.Pool, D.Morris, A.Hussain, and P.Ezra, "Improving radiation tolerance in e $2 \mathrm{v}$ CCD sensors," pp. http://www.e2v.com/e2v/assets/File/documents/imaging-space-and-scientificsensors/Papers/Improving\%20radiation\%20tolerance\%20in\%20e2v\%20CCD\%20sensors.pdf.

[2] C.J.Bebek and et.al., "Proton radiation damage in high resistivity n-type silicon CCDs," Proc. SPIE 4669, pp. 161-171, http://snap.lbl.gov/ccdweb/LBNL_49933.pdf, 2002. 
[3] N. J. Murray, D. J. Burt, D. Hall, and A. D. Holland, "The relationship between pumped traps and signal loss in buried channel CCDs," Proc. SPIE 8860, p. 88600H, 2013.

[4] I.V.Kotov, A.I.Kotov, J.Frank, P.O'Connor, V.Perevoztchikov, and P.Takacs, "CCD Base Line Subtraction Algorithms," IEEE Trans. Nucl.Science 57, No.4, pp. 2200 - 2204, 2010.

[5] N. J. Mostek, C. J. Bebek, A. Karcher, W. F. Kolbe, N. A. Roe, and J. Thacker, "Charge trap identification for protonirradiated p+ channel ccds," Proc. SPIE 7742, p. 774216, 2010. 